\title{
Effect of high- and low-pressure circulation patterns above mid-latitudes on the incidence of SARS-COV- 2 across wider European sector during early spring 2020
}

\section{Marek Kučera ( $\square$ marekkucera556@gmail.com )}

Charles University in Prague https://orcid.org/0000-0002-7798-3581

Athanasios Kyriazopoulos

Chief Translator at Radnet Editions, 23 Mousson Str., 11524 Nea Philothei, Athens

Peter Kučera

Radiodetection, Western Dr, Bristol BS14 OAF, United Kingdom

\section{Research Article}

Keywords: SARS-COV-2, weather, correlation, Europe, spring 2020

Posted Date: May 14th, 2020

DOI: https://doi.org/10.21203/rs.3.rs-28718/v1

License: (c) (1) This work is licensed under a Creative Commons Attribution 4.0 International License.

Read Full License 


\section{Abstract}

The presented paper evaluates SARS-CoV-2 in relation to high-, neutral and low- pressure zones above mid-latitudes (North Atlantic Oscillation/NAO and Arctic Oscillation/AO phases) and simultaneous effects of 8 meteorological elements across Europe and its wider region during early spring 2020. Data of national daily incidence of SARS-CoV-2 (for 86 countries and dependent territories) were correlated with daily values of 8 meteorological elements in 137 representative cities for 3 periods before an incidence day corresponding to incubation time of SARS-CoV-2. Period 0-9th day represented negative, period 514th day neutral and period 10-19th day positive NAO/AO pattern. Incubation time in last period was shifted, because some cases were linked to longer waiting for results of testing or in later course of SARSCoV-2. Patterns linked with high-, neutral and low- pressure zones above mid-latitudes were outlined. Results have shown that, during the high-pressure pattern above mid-latitudes, lower precipitation, higher pressure, weaker wind, higher sunshine, higher diurnal temperature range (higher maximum and lower minimum temperatures) were very unfavorable for incidence of SARS-CoV-2 (more cases) and that, during the low-pressure pattern above mid-latitudes, lower pressure, higher wind and less than NAO+ lower precipitation, higher sunshine and higher diurnal temperature range (mainly higher maximum temperatures) were unfavorable. For comparison these results were outputted with circulation conditions NCEP/NCAR reanalysis. During NAO+, precipitation and wind/minimum temperatures, precipitation and pressure; during NAO-, minimum temperatures, wind and sunshine/maximum, minimum and average temperatures, precipitation, sunshine and snow cover have the most simultaneous same/opposite effects.

\section{Introduction}

The 2019-20 coronavirus pandemic is an ongoing pandemic, caused by severe acute respiratory syndrome coronavirus 2 (SARS-CoV-2). The outbreak was identified in Wuhan, China, in December 2019, declared to be a Public Health Emergency of International Concern on 30 January 2020, and recognized as a pandemic on 11 March 2020 (WHO, 2020a, b).

The usual incubation period (the time between infection and symptom onset) ranges from one to 14 days; it is most commonly five days (lacobucci, 2020).

The aim of this study is to give a comprehensive picture of the 8 meteorological elements' effect on the incidence of SARS-CoV-2 during the main circulation patterns in the Euro-Atlantic area, in the northern hemisphere respectively (NAO-/AO- versus $\mathrm{NAO}+/ \mathrm{AO}+$ ). The goal is to detect significant differences between pre-dominant high pressure phase above mid-latitudes ( $\mathrm{NAO}+/ \mathrm{AO}+)$ and pre-dominant low pressure phase above mid-latitudes (NAO-/AO-) for better understanding the effect of main circulation patterns on the next behavior of SARS-CoV-2. 


\subsection{Recent studies about effect of weather on the incidence SARS- CoV-2}

SARS-COV -2 is a relatively large virus with a size of about $1-2 \mathrm{~nm}$ from a group of enveloped virus family with a positive-sense single-stranded RNA. The virus is transmitted by coughing and sneezing through direct contact with the infected people (respiratory droplets) and infected surfaces (can survive for hours outside the host). SARS-CoV-2 general signs and symptoms are fever, cough and breath problems. In severe cases, infection evolves to pneumonia, serious respiratory problems and, on rare occasions, it can be deadly, mainly for older people (Harmooshi et al., 2020).

While influenza virus is linked to cold weather and peak of flu season is reached in the middle of winter, it is still a question how SARS-CoV-2 is linked to weather. Widespread testing and dependence between cooler countries in the north may cause, that number of confirmed SARS-CoV-2 cases between cooler northern and warmer-humid southern regions is different. However, more southern countries have realized large-scale testing and the number of positive SARS-CoV-2 cases (per capita) are lower than in northern climate (Bukhari and Jamel, 2020).

Sajadi et al. (2020) predict Potential Spread in connection with Seasonality for SARS-CoV-2, using climate data from cities with prominent spread of SARS-CoV-2. Authors predict where are the most likely higher risks of spreading of SARS-COV-2. To date, SARS-CoV-2, has created a significant community spread in big cities in the countries along a narrow west east regions crudely along the $30-50^{\circ} \mathrm{N}$ latitude with similar weather conditions (average temperatures of $5-11^{\circ} \mathrm{C}$, absolute humidity $(4-7 \mathrm{~g} / \mathrm{m} 3$ ) and low specific (3-6 g/kg)). Significant predictors for spreading of a virus are mainly population proximity and interaction of people through travel.

Tang et al., 2009 have researched the survival of the airborne human coronavirus HCV/229E. This virus was used by authors as a representative or surrogate of respiratory coronary viruses and it was examined under various temperature and relative humidity conditions. Authors demonstrated that under such conditions, the virus has a half-life of 27 / 67 / only 3 hours at 30\% / 50\% / 80\% humidity. Here is an evidence, that near higher relative humidity, half-life of virus has considerably shorter time of survival. When the temperature drops as $6^{\circ} \mathrm{C}, \mathrm{HCV} / 229 \mathrm{E}$ half-life is redoubled to 6 hours at $80 \%$ humidity. Similar results have been found for seasonal flu (Lowen and Steel, 2014). It is assumed that this severe effect on prolonging the virus's half-life is linked to the spreading of the SARS-CoV-2 during low-temperature and high-humidity pattern of weather (Harmooshi et al., 2020).

Wang et al. (2020) researched the influence of air temperature and humidity on the transmission of SARS-CoV-2. Meteorological data for 100 Chinese cities with more than 40 cases were used. According to the study, the arrival of humid summer season could significantly diminish the transmission of the SARS-CoV-2.

Ma at al. (2020) explored the association between SARS-CoV-2 deaths and weather parameters (temperature and humidity) in 2299 SARS-CoV-2 related deaths. Both meteorological elements are 
considered as important factors for increasing/decreasing SARS-COV-2 mortality.

Chan et al. (2020) investigated on the effect of temperature and humidity on SARS-CoV-2. Authors used as target group of viruses HKU39849 strain. Facilitations of SARS-CoV-2 transmission were linked with lower temperature and dry conditions.

Xiao et al. (2020) found that big air pollution also has an effect on the impacts of SARS-CoV-2. An increase of only $1 \mu \mathrm{g} / \mathrm{m} 3$ in PM2.5 brings a $15 \%$ increase in the SARS-CoV-2 death rate at the level of significance 0.05 . Results are statistically significant and robust to secondary and sensitivity analyses.

Using data on local transmissions until the 23rd of March 2020, Araujo and Naimi (2020) in a preprint article developed an ensemble of 200 ecological niche models to project monthly variation in climate suitability for spread of SARS-CoV-2 throughout a typical climatological year. Although cases of SARSCoV-2 are reported all over the world, most outbreaks demonstrate a pattern of clustering in cooler and drier regions. The predecessor SARS-CoV-1 was linked to similar-dry and cold climate conditions. Authors expect asynchronous seasonal global outbreaks unless current trends of spreading SARS CoV-2 will be continuing. Models have shown that temperate cold and humid climates are more favorable to spread of the virus, whereas tropical hot and arid climates are less favorable. Nevertheless, model uncertainties are still high in the regions of sub-Saharan Africa, Latin America and South East Asia. Unsuitable climates can cause quick destabilization and reducing capacity of the virus.

Correspondence of Brassey et al. (2020) suggests that cold and dry conditions may influence the transmission of SARS-CoV-2. Authors gave a summary of evidences from unpublished papers till 22. March 2020:

- A cross-sectional study found that every $1^{\circ} \mathrm{C}$ of increase in the minimum temperature led to a decrease in the cumulative number of cases by 0.86 .

- A modeling study suggested a transitory reduction of incidence in spring and summer period and subsequently increase during the winter period 2020-2021.

- Another modeling study found that the current spread preferences are cool and dry conditions. 2002-3 SARS-CoV-1 outbreak was linked to similar weather conditions.

Conclusions and predictions of these studies have high uncertainty (their results are potentially biased because of uncontrolled confounding).

- Next two studies announce that daily mortality of SARS-CoV-2 is positively correlated with diurnal temperature range (DTR) but negatively correlated with relative humidity. Temperature and relative humidity are likely to contribute a maximum of $18 \%$ of the variation in transmission. Every $1^{\circ} \mathrm{C}$ increase in temperature / $1 \%$ increase in relative humidity lowered the $\mathrm{R}$ by $0.0383 / 0.0224$. 
- A very recent modeling study investigated the relationship between temperature and predicted the number of cases. Lower temperatures were worse for incidence of SARS-CoV-2 than were higher ones.

Lin et al. (2006) investigated to identify factors involved in the emergence, prevention and elimination of SARS-CoV-1 in Hong Kong from 11 March to 22 May 2003. A structured multiphase regression analysis has shown the potential effects of weather, time and interaction effect of hospital infection on severe acute respiratory syndrome. In colder days during the epidemic, the risk of increased daily incidence of SARS-CoV-1 was 18.18-fold (5.6-58.8 at the level of statistical significance 0.05 ) higher than in warmer days. Naturally decrease during the epidemic might by an average of 2.8 the total daily new cases every 10 days. The authors considered SARS-CoV- 1 transmission to be dependent on temperature changes during the climate year and on the multiplicative effect of hospital infection. SARS-CoV-1 retreated naturally during the further development of the epidemic.

Tan et al. (2005) have found a significant correlation between the SARS-CoV-1 cases and the air temperature seven days before the incidence and the seven-day time lag corresponded well with the wellknown incubation period. The optimum air temperature linked with the SARS-CoV- 1 cases (and its encourage virus growth) was from 16 to $28^{\circ} \mathrm{C}$. A sharp decrease (and too rise) of air temperatures related to the cold spell led to an increase of the SARS-CoV-1 cases - human immune system is influenced by cold weather. There are some evidences that a higher possibility for SARS-CoV-1 to reoccur is during the spring more than the autumn / winter.

Gardner et al. (2019) studied a background of Middle East respiratory syndrome coronavirus (MERS-CoV) from January 2015 to December 2017. To find linkages between primary MERS-CoV cases and previous weather conditions within the incubation period (2 weeks) in Saudi Arabia a case-crossover design using invariable conditional logistic regression was used. The full case dataset (1191 cases) was reduced to representative group most likely to represent spillover transmission from camels (446 cases). In research, meteorological data from localities closest to the largest city for each Saudi region were used (daily maximum, minimum and mean temperature (in ${ }^{\circ} \mathrm{C}$ ), relative humidity (in \%), wind speed (in $\mathrm{m} / \mathrm{s}$ ), and visibility (in $\mathrm{m}$ ). Lowest temperature (Odds Ratio $=1.27 ; 1.04-1.56$ at the level of statistical significance 0.05 ) and humidity (Odds Ratio $=1.35 ; 1.10-1.65$ at the level of statistical significance 0.05 ) were linked with incidence of MERS-CoV 8-10 days later, high visibility 7 days later (Odds Ratio $=1.26 ; 1.01-1.57$ at the level of statistical significance 0.05 ) and wind speed 5-6 days later.

Given the previous associations between viral transmission and humidity and temperature across which the majority of the SARS-CoV-2 cases have been observed until the present date, that lower humidity and lower temperatures are unfavorable for incidence of SARS-CoV-2. If SARS-CoV-2 is relatively sensitive to these types of weather, then it could be applied to optimize the SARS-CoV-2 mitigation strategies (Bukhari and Jamel, 2020). The provision of protective gear is also a very important factor for the prevention of SARS-CoV-2 infection. Other circulating viruses, such as flu copy a seasonal effect, and for that reason co-infection rates will have decline trend, which may have effect on death rate (Brassey et al., 2020). 


\subsection{Main circulation patterns in the mid-latitudes (North Atlantic Oscillation/NAO and Arctic Oscillation/AO phases)}

Seasonal climatic anomalies manifest themselves over large geographical regions. Some areas appear cooler and drier, whereas others, hundreds to thousands of kilometers away, warmer and wetter. These parallel structures with opposite phases and manifestations in climatology are referred to as teleconnections. Most often, they are determined using one point correlation maps, comparing the correlations of the height of the grid field points of the pressure level of $500 \mathrm{hPa}$ and the reference point. Low-frequency oscillations are also reflected in the ground pressure field. After depicting air pressure anomalies at sea level, the ground air pressure dipole is visible (Hurrell et al., 2003). A total of 13 modes have been identified that affect the northern hemisphere climate. The NAO (AO) mode is the only one of all modes having expression in Europe throughout the year, the other 3 main modes - EA, EU2 and EU1, are only visible in selected seasons. Among teleconnections we can include Southern Oscillation (ENSO) too, but it has no significant manifestations in Europe, it is considered with $\mathrm{AO}$ to be the most extensive oscillation affecting the climate on Earth.

The magnitude of the pressure gradient between the Arctic and moderate latitudes is closely related to the evolution of the polar vortex and the nature of the jet stream. In meteorology, this magnitude is linked with two major oscillation modes occurring above the northern hemisphere: Arctic Oscillation (AO) and North Atlantic Oscillation (NAO). AO is clearly identifiable in pressure fields (especially in winter) throughout the northern hemisphere, while the NAO is its equivalent to the Euro-Atlantic area. $90 \%$ of the oscillations are interconnected. If the $\mathrm{AO}$ and NAO indices both show negative values (negative phase), this results in a weaker and less pronounced polar vortex and a weaker and meandering jet stream. Stronger polar vortex and more zonal running and strong jet the stream, on the other hand, is associated with the positive phase of $\mathrm{AO}$ and NAO. Each phase has its typical manifestations in pressure, temperature and precipitation fields in individual regions, including Europe (Hurrell, 1995, Hurrell and Desser, 2009; Hurrell a van Loon, 1997; Kapala et al., 1998; Wanner et al., 2001).

The NAO describes in a meridional direction the transfer of atmospheric masses between the subtropical region in the Azores Pressure Area and the sub-polar in the Icelandic Pressure Area. It affects both seasonal and interdecadal variability in global circulation (Hurrell, 1995) and is the only oscillating mode to show statistically significant links with meteorological characteristics in Europe throughout the year. According to Hurrell et al. (2003) the area of influence of the NAO extends from the east of the USA to Siberia in the zonal and from the Arctic to the subtropical Atlantic in the meridional direction.

There are two types of situations: type A - equivalent of negative phase: NAO-/AO- (weakening of pressure formations in the North Atlantic) and type $B$ - equivalent of positive phase: $\mathrm{NAO}+/ \mathrm{AO}+$ (more developed pressure units). The distribution of pressure units over the Atlantic varies considerably in winter and summer - in the summer, the Azores High (the first NAO Action Center) dominates, which also extends northwards due to the shift of the intertropical convergence zone to the north. On the contrary, it weakens and shifts to the south during winter, with Icelandic Low dominating the Euro-Atlantic area (second NAO 
action center). Differences in air pressure deviations between the positive and negative NAO phases in action center areas are over $15 \mathrm{hPa}$. Significant changes in atmospheric circulation remodeling occur when the NAO index exceeds +1 or -1 (Hurrell, 1995).

During the positive phase of the NAO, anomalously higher air pressure occurs west to southwest of the coast of Europe in combination with anomalously low air pressure in the Arctic Atlantic. The flow generated by the rotation of pressure formations is intensified by a larger pressure gradient, causing a more pronounced western flow at moderate latitudes over (North) Atlantic and (Northern) Europe, a southern flow over the east coast of the US and north over western Greenland and Canada. The western flow brings warmer and damper sea air to Europe in winter and causes milder winter, while the northern flow from the Great Lakes to the north and northeast causes more waves of cold and blizzards. In the negative phase, the situation is reversed: the pressure formations above Iceland and the Azores are anomalously weakened (shallower Iceland low and less robust Azores high). A weak meridional pressure gradient causes a weak western flow, so much humid and warm ocean air does not get above Europe in winter, a suitable situation for meridional air mass transfer occurs and potential invasions of Arctic and Siberian air. With the extremely low oscillation index, seasonal Greenland High increases, bringing aboveaverage air temperatures in Canada, the United States and Greenland, but significant cold air inflows over Europe. The following patterns of correlation of the NAO index with the most important meteorological elements - precipitation, air temperature and pressure field distribution (Blade et al., 2012) were identified:

1.) Summer: During the positive phase of the $N A O$, there is a robust pressure high above the British Isles, extending from the Azores to Scandinavia, with a center in Scotland and Northern Ireland. There is a deep low-pressure area above Greenland, and a low-pressure field extends over the Mediterranean. On the contrary, during the negative phase of the NAO, anomalous high-pressure spreads over the area of Iceland and Greenland, and areas of mid- and subtropical latitudes from North America over the Atlantic to midlatitudes of Europe and the Mediterranean report negative surface pressure anomalies. The largest negative pressure anomalies are achieved in northern Germany and Denmark (Boé et al., 2008). Positive correlations with precipitation are achieved during the summer and positive NAO phases in the British Isles, Benelux, north of France, Germany and Poland to the Eastern Carpathians, the south of Scandinavia and the Baltic Sea. The robust high pressure above Northwest Europe during the NAO + (Action Center) causes a flow of cooler and drier air over the continent, which has a stabilizing effect on temperatures and precipitation in Central Europe. Negative correlations with precipitation have been confirmed across the Mediterranean, from Spain to Turkey, with the highest values in the Adriatic and the Balkan. The penetration of cooler air from the northwest generates numerous precipitations and low temperatures mainly above the Balkans, Italy, Turkey, to Israel and Egypt. On the contrary, the robust pressure above the British Isles brings, in addition to drought, above-average temperatures on the British Isles, and from west and north-west coasts of Europe to the Baltic. During the negative NAO phase, temperature and precipitation responses are reversed.

2.) Winter: The different nature of winters is largely related to the tracking of Atlantic cyclones in winter. During the winter when the polar vortex is locked above the sub-polar region and the zonal flow reaches 
high levels, the low-pressure action center is located more northerly on the Greenland - Iceland axis. The deep Icelandic low travel across Europe by northern tracks - over the British Isles and Scandinavia, at most the coast of the North Sea and the Baltic Sea. Europe (except the British Isles and Scandinavia) is on the front of the quasi-stationary deep low pressure zone in the warm, predominantly southwestern, during the winter NAO + phases, while stormtrack is shifting from the Island-British Isles axis to the north of Europe. The activity of Mediterranean cyclones is minimal - in the Mediterranean there is dry and relatively cold weather (thanks to clear anticyclonic nights). The northernmost and, in particular, the more robust regions of the Azores high reach western, southern but also central Europe more often than usual during these phases. During the negative phase the situation is reversed - the stormtracks are due to the extensive meanders of the polar and arctic front and weakened zonal air transport curved over the Mediterranean. Due to the disintegration of the polar vortex, the jet-stream is forced to the south, generating low pressure over lower latitudes (southern, moderate and subtropical regions). The low pressure over the Mediterranean and the high pressure over Scandinavia and the northern half of Europe, together with the more pronounced Siberian anticyclone, then create suitable conditions for invasions of Arctic or dry and cold Siberian air over Europe (the Mediterranean pressure lows "pulling" cold air towards them). Pressure field such this, typical for NAO-phases, is associated with cyclonic weather with higher temperatures and higher rainfall totals over the Mediterranean, and drier and cold conditions over the northern half of Europe. In winter, correlations of the NAO index with temperatures and precipitation have a stronger zonal pattern than in summer. A clear signature of the positive NAO phase on air temperatures, precipitation, as well as the total pressure field and flow is present from North America to eastern Siberia and is associated with overall warming in Siberia and cooling in eastern Canada and from subtropical to tropical latitudes.

\section{Data And Methods}

Three types of data are used in the analysis: daily meteorological data from International Exchange Network (covered by World Meteorological Organisation); daily incidence of SARS- $\mathrm{C}_{0} \mathrm{~V}-2$ measured by World Health Organization and NCEP/NCAR Reanalysis from Physical Sciences Laboratory, NOAA. We have focused to evaluate and quantify effect weather to SARS-CoV-2 for countries in wider European region. It comprises 86 countries and dependent territories and 137 cities in 13 main examined regions (Fig. and Table 1) in Europe, Africa, Asia and North America. All days for the period from 8. February to 7. April 2020 were filtered to whether they fall under compact period of incidence SARS-CoV-2, with starting day with minimally $25 \%$ of value of maximum incidence. Then was chosen the 3 periods from International Exchange Network for representative meteorological stations in these countries (overall 137 localities) and correlated with each other.

We used for this analysis meteorological data of: TG-average temperature $\left({ }^{\circ} \mathrm{C}\right), \mathrm{TX}$-maximum temperature $\left({ }^{\circ} \mathrm{C}\right), \mathrm{TN}$-minimum temperature $\left({ }^{\circ} \mathrm{C}\right), \mathrm{RR}$-24-hours precipitation $(\mathrm{mm}), \mathrm{PP}$-air pressure (Hpa), WW-average daily wind speed $(\mathrm{km} / \mathrm{h})$, SS-sunshine duration (h) and snow cover (cm). From pairs of daily health and previously meteorological data were computed Spearman correlation ranks. 
From these data were outlined correlation matrix and correlation maps (for every city, with significance at level 0.10 ) for every from 8 meteorological elements and 3 periods of incubation time. We were chosen 3 periods of an incubation time as an input of meteorological data to correlation analysis: 10-19 days before an incidence, 2 . 5-14 days before an incidence and 1, 3. 0-9 days before an incidence

Mean date of meteorological data before an incidence was for the $1^{\text {st }}$ period 16 . March, for $2^{\text {nd }}$ period 21. March and for $3^{\text {rd }}$ period 26. March. These dates were closer to end of incidence time series, because count of the stations/countries with longer outbreak was decreasing by time. Dates falling under interval of meteorological data before an incidence (middle position of 10 day window) was from 7. March to 22 . March for $1^{\text {st }}$ period, from 12. March to 27. March for $2^{\text {nd }}$ period and from 17. March too 2 . April 2020 for $3^{\text {rd }}$ period. (We neglected Bahrain station, where outbreak have begun very early $(25 \%$ threshold was reached in 26. February and meteorological data were used from date 8 . February-periods would be extended for 3.5 ago in comparison to results). These periods relative clearly fitted with strong NAO+/AO+ phase with NAO index over 1.0 during the $1^{\text {st }}$ period, neutral transient phase of NAO/AO during the $2^{\text {nd }}$ period and strong NAO- or relative strong $A O$ neutral phase with NAO index under -1.0 during the $3^{\text {rd }}$ period. In this positioning, effect main circulation phases above mid-latitudes to SARS-CoV-2 in 3 incubation time periods could be examined. Incubation time was in last period shifted, because some part of cases was linked with longer waiting for results of testing or in later course of SARS-CoV-2, therefore results could better fit to real incubation time before a day of results of tests.

For identified positive, negative and neutral phase of NAO/AO in outputs were computed NCEP/NCAR Reanalysis plots for verification and understanding of circulation conditions.

Spearman ranks were finally correlated to each other for findings of patterns, whose meteorological elements have a common effect on the incidence of SARS-CoV-2, with same shift or opposite shift. These computations were realized for 13 main regions.

\section{Results And Discussion}

In this chapter we evaluate the resulting nature of the spatial variability of effects 8 meteorological elements to incidence SARS-CoV-2 during 3 periods falling under incubation time and main circulation patterns. We also point to differences in incidence of SARS-CoV-22 between main circulation patterns across Europe and its wider area (especially differences between the positive and negative phases NAO / AO). On the basis of NCEP / NCAR Reanalysis we evaluate the affiliation to individual phases of NAO / AO. Finally, the simultaneous same or opposite effects of meteorological elements on the incidence SARS-CoV-2 during phases are displayed using a correlation matrix and a schematic map.

\subsection{Spatial distribution of effects of 8 meteorological elements on the incidence SARS-COV-2: evident impact of predominant mid-latitudes circulation patterns}


Firstly, paired Spearman correlation ranks between daily value of incidence of SARS-CoV-2 and 8 meteorological elements (TG, TX, TN, RR, PP, WW, SS, SC) were computed (Table 2). Correlation coefficients were tested at level of significance 0.10 . Then were outlined correlation maps for each element and 137 stations/86 countries and dependent territories (Fig. 2). Values for countries and dependent territories were derived as averages from results for stations.

Results of spatial distribution of correlations computed for sea level pressure (PP) and precipitation (RR) have shown, that a substantial part of computed outlined coefficients copy the main NAO/AO pattern, when the majority of cities in mid-latitudes match the main phases of NAO/AO (Blade et al., 2012; Wanner et al., 2001; Hurrell and Desser, 2009; Boé et al., 2008, Hurrell et al., 2003; Hurrell, 1995). Mainly during strong $\mathrm{NAO}+/ \mathrm{AO}+$ phase during $1^{\text {st }}$ incubation period (10-19 days before an incidence) were thanks to $A O$ and NAO index values above 1.0 were present strong positive/negative correlations $P P / R R$ with SARS-CoV-2 incidence above western Europe, British Islands, Central Europe, southern and middle parts of Scandinavia, Baltic and almost all eastern Europe except for Black sea region; and on the other hand significant negative/positive correlations in Mediterranean and too above tropical, the most southeast main regions (Fig. 2, PP and RR row and 10-19 days (NAO+) column). A part of investigated incidence of SARS-CoV-2 therefore was through the incubation period linked with predominant positive NAO/AO phase.

Situation during NAO-/AO- during the $3^{\text {rd }}$ period (or neutral $\mathrm{AO}$ for better description, because $\mathrm{AO}$ index wasn't in minus values as NAO index) had inverse and over mid-latitudes low pressure field was preserved. More PP with SARS-CoV-2 incidence positive correlations and on the contrary, pressure over Scandinavia was height, but not with origin of Azores height with warm air, but Greenland high with cold air (northerly from southerly-shifted stormtrack in this period), and part of tropical main regions had high pressure too (subtropical heights were pushed from north to the south), were observed. RR were evenly distributed over mid-latitude area.

Considering that the behavior of coronavirus replicates the behavior of NAO and AO, we are not only able to estimate how the ground-level meteorological elements will behave during the mid-latitude predominant low- and high-pressure phases. The following subchapters deal with the observed behavior of the effects of meteorological elements on the incidence of SARS-CoV-2 during the 3 basic modes of Euro-Atlantic and North-hemispherical circulation, respectively.

\subsubsection{SARS-CoV-2 during predominant anticyclonic weather (NAO+/AO+) in the mid-lattitudes}

As we have shown in Fig. 2 and Fig. 3, the entire column for 10-19 day (NAO+) shows signs of a strong $\mathrm{NAO}+/ \mathrm{AO}+$ phase with strong predominant high-pressure conditions over anticyclonic belt above midlatitudes. 
Results have shown that, during the high-pressure pattern above mid-latitudes, lower precipitation, higher pressure, weaker wind, higher sunshine, higher diurnal temperature range (higher maximum and lower minimum temperatures) were very unfavorable for incidence of SARS-CoV-2.

We can confirm previous findings, that higher drought or diurnal temperature range are very unfavorable for incidence SARS-COV-2, what means, that incidence SARS-CoV-2 is during this type of weather higher (Bukhari and Jamel, 2020; Sajadi et al., 2020; Wang et al., 2020; Ma et al., 2020, Chan et al., 2020; Araujo and Naimi, 2020; Brassey et al., 2020) and effect of drought can be devastating.

Results regarding dry conditions are very similar as for SARS-CoV-1 (Lin et al. (2006); Tan et al. (2005); Araujo and Naimi (2020); Brassey et al. (2020)) MERS-CoV (Gardner et al. (2019) and influenza or similar viruses' outbreaks (Harmooshi et al., 2020; Tang et al., 2009; Lowen and Steel, 2014).

We can hypothesizes how the weather impact on the incidence of SARS-CoV-2 is linked to mood in the population. Bassi (2013) concluded that bad weather caused worse mood in most of the population and is linked e.g. with lower interest for voter turnout in elections. Similarly, good mood was associated with lower humidity (Sanders \& Brizzolara, 1982), high levels of solar radiation (Parrot \& Sabini, 1990), high barometric pressure (Goldstein, 1972) and high temperatures (Cunningham, 1979). The effect of solar radiation was strongest in spring (Keller et al., 2005).

\subsubsection{SARS-COV-2 during weather with neutral circulation in mid- lattitudes}

During decline NAO index from values above 1.0 under -1.0 (neutral phase of NAO, AO respectively), are visible in Fig. 2 and Fig. 3 signs of beginning polar vortex drop-correlations PP with incidence SARSCoV-2 is even stronger such in NAO- equivalent above British Isles, area of Genoa cyclogenesis, Scandinavia and Baltic, but vortex is not dropped to the southern regions yet. Higher RR starts have less positive (or clearly negative) effect on incidence in Stormtrack Area and Mediterranean and SS and weaker wind starts have less negative effect. Higher temperatures and diurnal temperature range are not as bad as during NAO+/AO+. Cyclogenesis in Genoa area is strong, and RR can be more unfavorable than near NAO-.

\subsubsection{SARS-COV-2 during predominant cyclonic weather (NAO-/AO-, AO neutral respectively) in the mid-latitudes}

It appears that NAO-/AO- phases play an important part in the weakening effect of drought on the incidence of SARS-CoV-2 during spring, summer and autumn. Although in winter this weather is linked with big coldwaves and lower temperatures are significantly linked with higher incidence in Western, Central Europe and larger part of Iberian peninsula, precipitation, humidity and cloudiness which 
NAO-/AO-brings to mid- and subtropical latitudes have substantial effect on the decreasing of the number of diagnosed SARS-CoV-2 cases.

During the low-pressure pattern above mid-latitudes (Fig. 2 and Fig. 3, the entire column for 0-9 day (NAO-)), lower pressure, higher wind and less than NAO+ lower precipitation, higher sunshine and higher diurnal temperature range (mainly higher maximum temperatures) above mid-latitudes were unfavorable. The effect of negative PP is widely spread across all regions, except for parts of Arctic and Scandinavia (Greenland height), RR starting to be more unfavorable and higher SS and weaker WW more favorable with comparison with NAO+. SC is appearing in Europe and is linked with higher incidence (and cold weather(!)). Lower temperatures are more unfavorable for higher incidence of SARS-CoV-2, mainly in western half of Europe. We can therefore certify favorable effect of PP and temperatures on the virus' incidence both in Eastern half of Europe and eastern regions during NAO-/AO-.

On the pages https://www.cpc.ncep.noaa.gov/products/precip/CWlink/pna/nao.shtml and https://www.cpc.ncep.noaa.gov/products/precip/CWlink/daily_ao_index/ao.shtml we can monitor daily evolution of NAO and AO index. There are available 16-day predictions of indices, which can help to estimate the impact of main phases of circulation conditions on increase/decrease of incidence SARS$\mathrm{C}_{0} \mathrm{~V}-2$ during seasons in 2020 and next years. Evolution of NAO and AO index during winter 2019/2020 and early spring 2020 contains Fig. 3. Investigated 3 periods of incubation time during early spring 2020 and 16-day prediction are marked here.

Regarding the findings of Bukhari and Jamel, 2020; Sajadi et al., 2020; Wang et al., 2020; Ma et al., 2020, Chan et al., 2020; Araujo and Naimi, 2020; Brassey et al., 2020 we are suggesting that the observed unfavorable cooling effect of temperature on the incidence of SARS-CoV-2 can be weakened by better mood and more spending leisure time outside during the sunny, warm, anticyclonic weather (Bassi, 2013; Sanders \& Brizzolara, 1982; Goldstein, 1972; Parrot \& Sabini, 1990 and Cunningham, 1979). This effect can be amplified by strong solar radiation in the spring (Keller et al., 2005). This effect can be especially strong in the case of SARS-CoV-2, when pandemics is widely spread, and people have to carefully choose when they can afford to use their time to stay outdoors.

\subsection{NCEP/NCAR Reanalysis comparison with 3 incubation time periods}

Computed NCEP/NCAR Reanalysis plots for identified positive, negative and neutral phase of NAO/AO during incubation time periods in Fig. 2 and 3 were verified and plotted for better understanding of circulation conditions of period before substantial outbreak of SARS-CoV- 2 .

In Fig. 4 we can refer to conditions during individual 3 phases of incubation time/NAO. At maps of H500 we can see weakening of polar vortex (from NAO+ to NAO- phase)-Azores height and Icelandic Low both. Domain of low pressure above Arctic is weakened and low geopotential is dropped to Mediterranean. RH850 shows dropped zone of higher relative humidity from northern mid-latitudes during 
$\mathrm{NAO}+$ to Mediterranean during NAO-, while in northern regions relative humidity decreased. T850 immediately responds to drop of polar vortex and zone with lower temperatures fast covers mid-latitudes in Europe and parts of Mediterranean. Zonal and meridional flow (Uwinds an Wwinds) are weakened across northern and southern latitudes both.

\subsection{Simultaneous same and opposite effects of meteorological elements}

Finally, we have made correlation matrixes between effects of 8 meteorological elements on the incidence of SARS-COV-2, every with each other. This output is computed for 13 partial regions, separately (Table 3). Relationships significant at level 0.10 were given to the maps (Fig. 5).

We can confirm bigger connections between variables (Table 3) derived from Table 2 in the most affected main regions with the biggest rate of statistically significant responses-mainly Western Europe Western Mediterranean and Balkan, Arctic and Central Europe too. In the regions, where pandemics started to spread later, or incidence rates were low (mainly southern tropical, or Asian regions, Eastern Europe eventually) are relationships between variables and overall amount of statistically significant responses is lower.

It is interesting that in Arctic almost all variables are linked with same simultaneous shift, while in Western Europe, Western Mediterranean and Balkan were identified relative strong opposite simultaneous shifts of variables. It shows that in Arctic (or the most northern regions) could be conditions for outbreaks of SARS-CoV-2 relatively fast improved or worsened, while in southern regions are working negative feedbacks trying to autoregulate and stabilize conditions of spreading of virus. Conditions in Arctic can be linked with Arctic Amplification feedback and general shift of all meteorological elements the same way (Budikova, 2009; Petouhkov a Semenov, 2010, Francis a Vavrus, 2012, Walsh, 2014 and others).

It appears that in the same shift act PP and SS with TG; PP, SS and WW with TX; PP, RR and WW with TN -mainly during NAO- phase; temperatures, WW and SC with RR-mainly during NAO+ phase; temperatures and SS with PP; temperatures, SS and RR during NAO+/NAO- phases both strongly; temperatures, WW and PP with SS-mainly during NAO- phase and WW, RR with SC. Opposite simultaneous effect is more common in favor NAO- phase with blue NAO- signs: red NAO+ symbols on Fig. 5 ratio 36:24.

It appears totally averse effect act RR, PP, WW and SC with TG and TX-mainly during NAO-phases; PP, WW and SS with TN-during NAO+ and NAO- phases both; temperatures, PP, WW and SS with RR during $\mathrm{NAO}+$ and NAO- both; temperatures, RR and WW with PP-mainly during NAO+; temperatures, RR, PP, SS with WW; temperatures, RR, WW and SC with SS-mainly during NAO-phases and temperatures, PP and SS with SS-mainly during NAO- phases. Opposite simultaneous effect is more common in favor NAOphase, again with of NAO-: NAO+ symbols on Fig. 5 ratio 47:29. 
This means that during NAO- are relationships between variables stronger and that opposite effects are more common than same effects, what can lead to autoregulation.

\section{Conclusions}

This analysis provides evidence of an environmental component contributing to the development of SARS-COV-2 infections. We investigated the effect of 8 meteorological elements on the incidence of SARS-COV-2 during 3 periods of incubation time of the virus corresponding with main circulation patterns in mid-latitude areas in Europe and its wider region during early spring 2020.

Results suggest that incidence of SARS-COV-2 can depends on main circulation patterns in Euro-Atlantic or Northern Hemisphere region, respectively, linked with high-, neutral and low- pressure zones above midlatitudes. Results have shown that, during the high-pressure pattern above mid-latitudes, lower precipitation, higher pressure, weaker wind, higher sunshine, higher diurnal temperature range (higher maximum and lower minimum temperatures) were very unfavorable for incidence of SARS-CoV-2 (more cases) and that, during the low-pressure pattern above mid-latitudes, lower pressure, higher wind and less than NAO+ lower precipitation, higher sunshine and higher diurnal temperature range (mainly higher maximum temperatures) were unfavorable. For comparison these results with circulation conditions NCEP/NCAR reanalysis were outputted.

For better understanding, simultaneous effects of meteorological elements were investigated. During $\mathrm{NAO}+$, precipitation and wind/minimum temperatures, precipitation and pressure; during NAO-phase minimum temperatures, wind and sunshine/maximum, minimum and average temperatures, precipitation, sunshine and snow cover have the most simultaneous same/opposite effects.

We agree with previous findings suggesting that incidence SARS-CoV-2 is higher during drought (and low humidity and precipitation) or higher diurnal temperature range (Bukhari and Jamel, 2020; Sajadi et al., 2020; Wang et al., 2020; Ma et al., 2020, Chan et al., 2020; Araujo and Naimi, 2020; Brassey et al., 2020). Results regarding dry conditions are very similar as for SARS-CoV-1 (Lin et al. (2006); Tan et al. (2005); Araujo and Naimi (2020); Brassey et al. (2020)) MERS-CoV (Gardner et al. (2019) and influenza or similar viruses' outbreaks (Harmooshi et al., 2020; Tang et al., 2009; Lowen and Steel, 2014). We have added to the list of unfavorable meteorological elements the following options.

The weather impact on the incidence of SARS-CoV-2 can be linked to mood in the population (Bassi, 2013). Mainly NAO+ phases are associated with lower humidity (Sanders \& Brizzolara, 1982), high levels of solar radiation (Parrot \& Sabini, 1990), high barometric pressure (Goldstein, 1972) and high temperatures (Cunningham, 1979). The effect of solar radiation was strongest in spring (Keller et al., 2005), which can negatively affect incidence in April and May 2020. We are suggesting that the observed unfavorable cooling effect of temperature on the incidence of SARS-CoV-2 can be weakened by better mood and more spending leisure time outside during the sunny, warm, anticyclonic weather. 
During NAO+, mainly precipitation and wind/minimum temperatures, precipitation and pressure; during NAO- phase minimum temperatures, wind and sunshine/maximum, minimum and average temperatures, precipitation, sunshine and snow cover have the most simultaneous same/opposite effects.

\section{References}

1. Araujo MB., Naimi B (2020) Spread of SARS-CoV-2 Coronavirus likely to be constrained by climate medRxiv 20034728 https://doi.org/10.1101/2020.03.12.20034728.

2. Bassi A (2013) Weather, Mood, and Voting: An Experimental Analysis of the Effect of Weather Beyond Turnout A Bassi SSRN 2273189.

3. Blade I, Liebmann B, Fortuny D (2012) Observed and simulated impacts of the summer NAO in Europe: implications for projected drying in the Mediterranean region 10.1007/s00382-011-1195-x.

4. Boé J, Terray L, Cassou C, Najac J (2008) Uncertainties in European summer precipitation changes: role of large scale circulation Dyn. 10.1007/s00382- 008-0474-7.

5. Budikova D (2009) Role of Arctic sea ice in global atmospheric circulation: A review. Global Planet. Change 68(3).

6. Bukhari Q and Jameel Y (2020) Will Coronavirus Pandemic Diminish by Summer? SSRN: https://ssrn.com/abstract=3556998 or http://dx.doi.org/10.2139/ssrn.3556998.

7. Cunnigham M R (1979) Weather, Mood, and Helping Behavior: Quasi-experiments with Sunshine Samaritan, Journal of Personality and Social Psychology 37 1947-1956.

8. Chan KH, Peiris JS, Lam SY, Poon LL, Yuen KY, Seto WH. (2020). The Effects of Temperature and Relative Humidity on the Viability of the SARS Coronavirus. Adv Virol. 2011: p. 734690.

9. Francis JA, Vavrus SJ (2012) Evidence linking Arctic amplification to extreme weather in midlatitudes. Geophys. Res. Lett. 39 L06801 10.1029/2012GL051000.

10. Gardner EG, Kelton D, Poljak Z, Van Kerkhove M, von Dobschuetz S, Greer AL (2019) A case-crossover analysis of the impact of weather on primary cases of Middle East respiratory syndrome. BMC Infect. Dis. 19 (1), 113. https://doi.org/10.1186/ s12879-019-3729-5.

11. Goldstein KM (1972) Weather, Mood, and Internal-External Control Perceptual Motor Skills 35786.

12. Harmooshi N, Shirbandi K, Rahim F (2020) Environmental Concern Regarding the Effect of Humidity and Temperature on SARS-COV-2 (SARS-COV-2) Survival: Fact or Fiction. SSRN: https://ssrn.com/abstract=3563403 or http://dx.doi.org/10.2139/ssrn.3563403.

13. Hurrell JW (1995) Decadal trends in the North Atlantic oscillation: Regional temperatures and precipitation. Science 269 676-679.

14. Hurrell JW, Kushnir Y, Ottersen G, Visbeck M (2003) An overview of the North Atlantic Oscillation. In: The North Atlantic Oscillation: Climatic Significance and Environmental Impact, Geophysical monograph 134. Washington, DC: American Geophysical Union 2003 p. 1-35

15. Hurrell JW, Desser C (2009) North Atlantic climate variability: The role of the North Atlantic Oscillation 10.1016/j.marsys.2008.11.026 
16. Hurrell JW, Van Loon H (1997) Decadal variations in climate associated with the North Atlantic Oscillation. Clim. Change 36, 301- 326.

17. lacobucci G (2020) 60 seconds on ... anosmia [loss of smell]. BMJ. 368: m1202. doi:10.1136/bmj.m1202. PMID 32209546

18. Kapala AH, Machel H, Flohn H (1998) Behaviour of the centres of action above the Atlantic since 1881. Part II: Associations with regional climate anomalies. Int. J. Climatol. 18 23-36.

19. Keller MC, Fredrickson BL, Ybarra O, Côté S, Johnson K, Mikels J, Conway A, Wager T (2005). A Warm Heart and a Clear Head. The Contingent Effects of Weather on Mood and Cognition Psychological Science. 16(9) 724-731.

20. Lin K, Yee-Tak Fong D, Zhu B, Karlberg J (2006) Environmental factors on the SARS epidemic: air temperature, passage of time and multiplicative effect of hospital infection. Epidemiol Infect 134 223 (2006).

21. Lowen AC and Steel $J(2014)$. Roles of humidity and temperature in shaping influenza seasonality. $J$ Virol 2014 88(14): p. 7692-5.

22. Ma Y, Zhao Y, Liu J, He X, Wang B, Fu S, Yan J, Niu J, Luo B (2020) Effects of temperature variation and humidity on the death of SARS-COV-2 in Wuhan, China. Science of The Total Environment 2020: p. 138226.

23. Parrot GW, Sabini J (1990) Mood and Memory under Natural Conditions: Evidence for Mood Incongruent Recall. Journal of Personality and Social Psychology.

24. Petoukhov V, Semenov V (2010) A link between reduced Barents-Kara sea ice and cold winter extremes over northern continents. J. Res.-Atmos. 115 (2010).

25. Sajadi, MM, Habibzadeh P, Vintzileos A, Shokouhi S, Miralles-Wilhelm F, Amoroso A (2020) Temperature, Humidity and Latitude Analysis to Predict Potential Spread and Seasonality for SARSCOV-2. SSRN, 2020.

26. Sanders J L, Brizzolara MS (1982) Reand Mood. Journal of General Psychology 107 155-156.

27. Tang JW (2009) The effect of environmental parameters on the survival of airborne infectious agents. J R Soc Interface 2009, 6 Suppl 6: p. S737-46.

28. Ten J, Mu L, Huang J, Yu S, Chen B, Yin J (2005) An initial investigation of the association between the SARS outbreak and weather: with the view of the environmental temperature and its variation. Journal of Epidemiology and Community Health 59 186-192.

29. Walsh JE (2014) Intensified warming of the Arctic: Causes and impacts on middle latitudes, Global and Planetary Change Volume 117.

30. Wang, J, Tang K, Feng K, Weifeng L (2020) High Temperature and High Humidity Reduce the Transmission of SARS-COV-2. SSRN 2020.

31. Wanner H, Bronninmann S, Casty C, Gyalistras D, Luterbacher J, Schmutz Ch, Stephenson D B, Xoplaki E (2001) North Atlantic oscillation - Concepts and studies, Surveys in Geophysics. 22: 321382, 2001. 
32. WHO (2020a) Statement on the second meeting of the International Health Regulations (2005) Emergency Committee regarding the outbreak of novel coronavirus (2019-nCoV). World Health Organization. 30 January 2020. Archived from the original on 31 January 2020. Retrieved 30 January 2020.

33. WHO (2020b) WHO Director-General's opening remarks at the media briefing on SARS-COV-2-11 March 2020. World Health Organization. 11 March 2020. Retrieved 11 March 2020.

34. Xiao W, Nethery RC, Sabath BM, Braun D, Dominici D (2020) Exposure to air pollution and SARS-COV2 mortality in the United States. medRxiv 2020.04.05.20054502; doi:

https://doi.org/10.1101/2020.04.05.20054502

\section{Declarations}

Funding: No funding was received.

Conflicts of interest/Competing interests: The authors declare that they have no conflict of interest.

Consent for publication: The authors agree with publication in Air Quaity, Atmosphere \& Health (2020) journal.

Availability of data and material: Meteorological data are available on International Exchange Network (e.g. meteomanz.com - http://www.meteomanz.com/index) and Health data were provided by XIstat (MS Excel extension) COVID19 Package built in the program. Reanalyses were plotted from NCEP/NCAR Reanalysis tool at the page https://psl.noaa.gov/data/gridded/tables/daily.html.

\section{Tables}

Due to technical limitations, Tables 1-3 are provided in the Supplementary Files section.

\section{Figures}




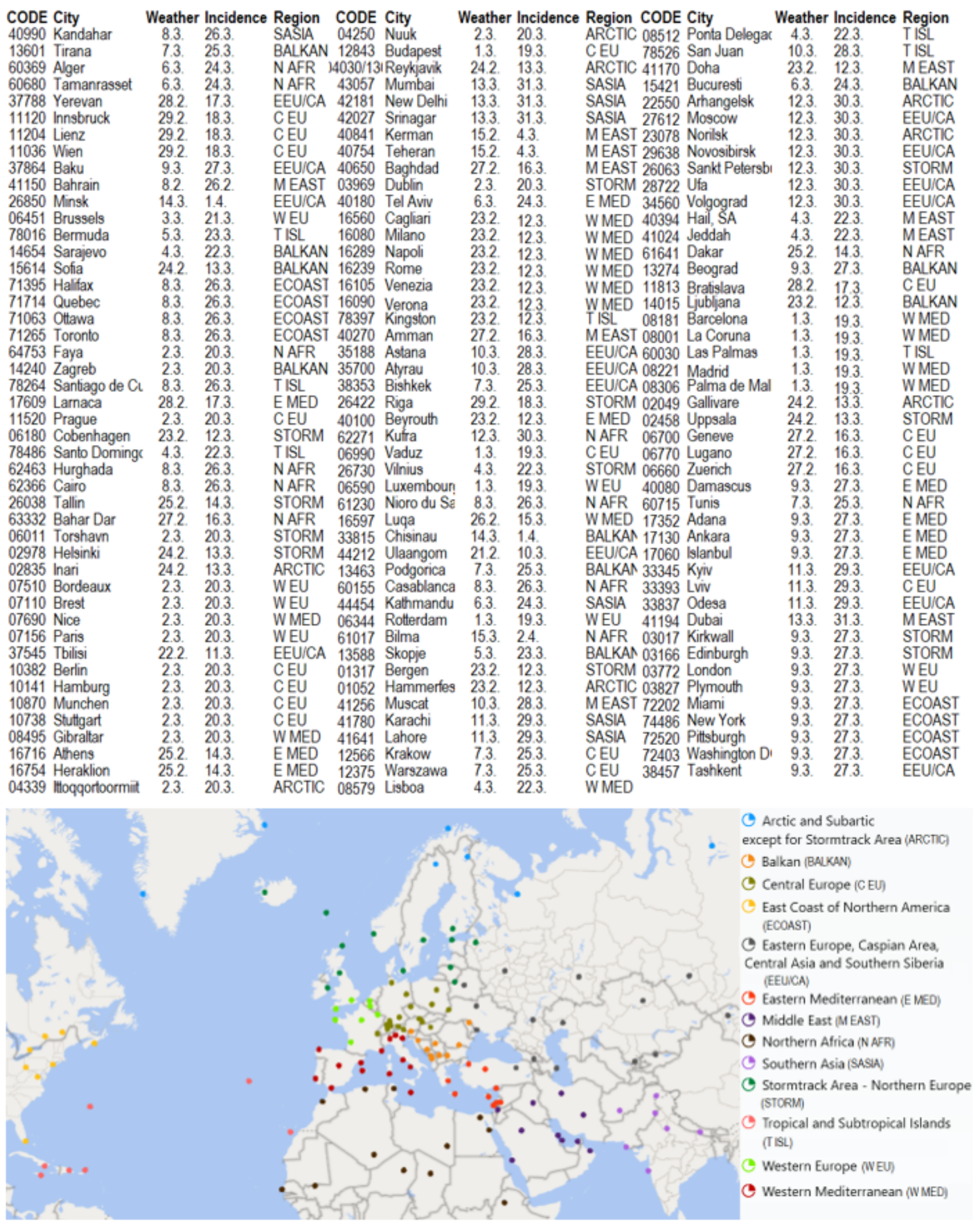

\section{Figure 1}

Up: List of 137 cities (representative meteorological stations) with their WMO CODE, date of the first day of meteo data (Weather) and incidence data (Incidence) entering to the computations and main region (Region) of cities; Down: Map of used stations (cities) within 13 main regions 


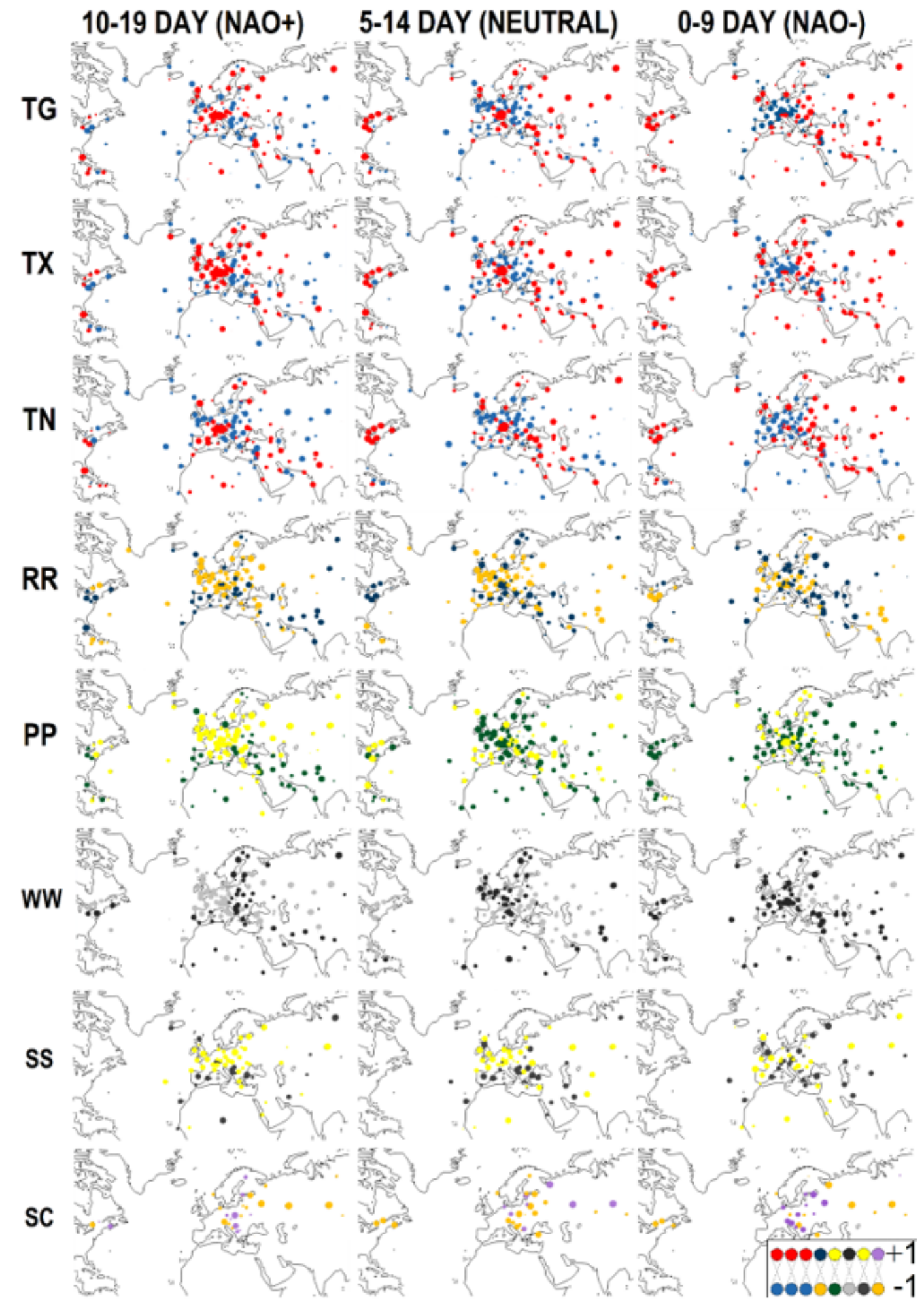

Figure 2

A: Size of correlation coefficients from Table 2 for every meteorological element (same as Table 2) 

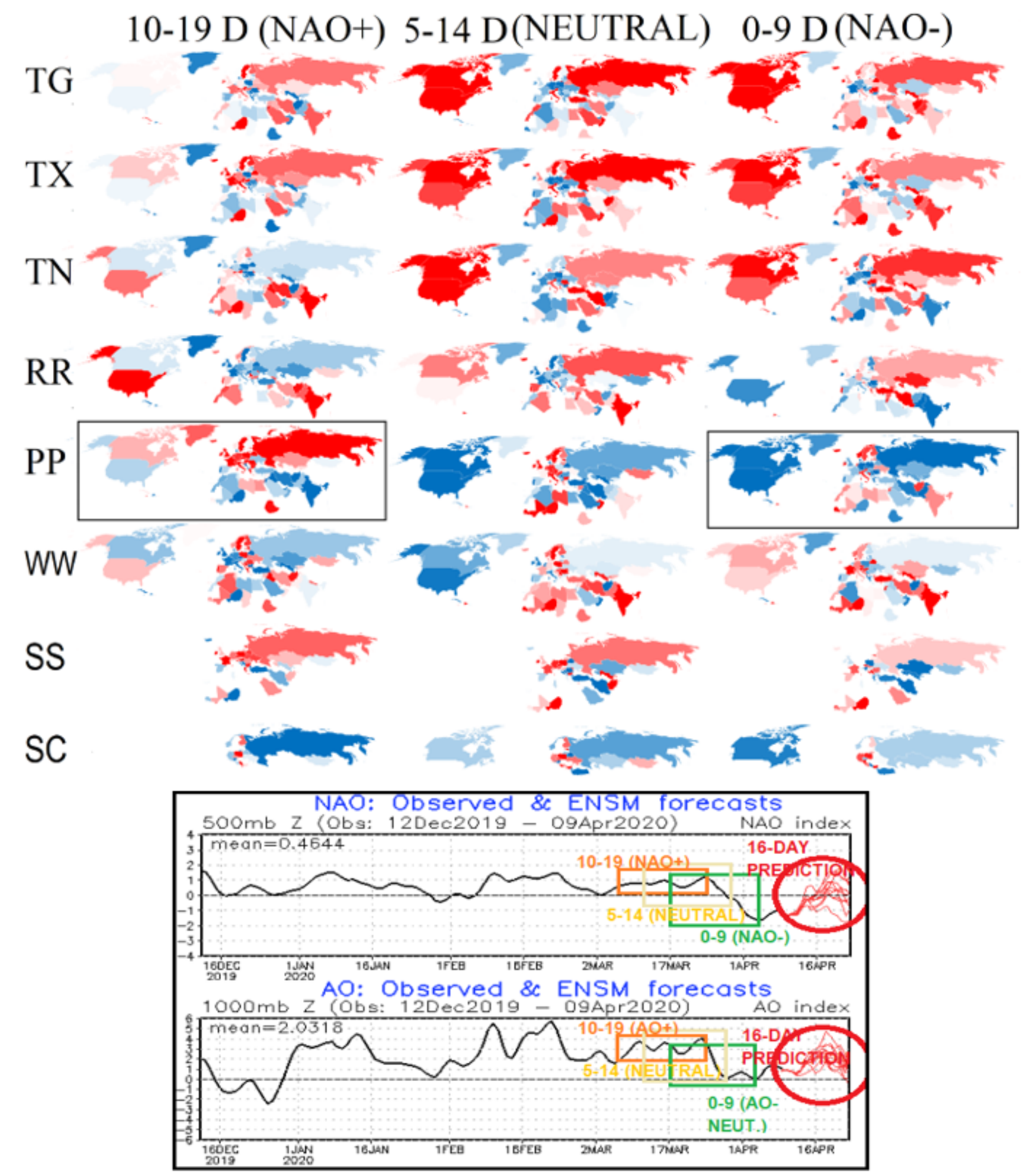

\section{Figure 3}

Up: Average correlation coefficients from Table 2/Fig. 2 for all countries and dependent territories. In a rectangle averages for PP during 1st period of incubation time (0-9 days before an incidence) corresponding to NAO-/AO- or AO neutral phase respectively an 3rd period of incubation time (10-19 days before an incidence) corresponding to NAO+/AO+ phase (Basemap (c) Geonames, MSFT); Down: NAO 
and $A O$ index during winter 2019/2020 and early spring 2020. Investigated 3 periods of incubation time during early spring 2020 are marked with rectangles, prediction with red circle.
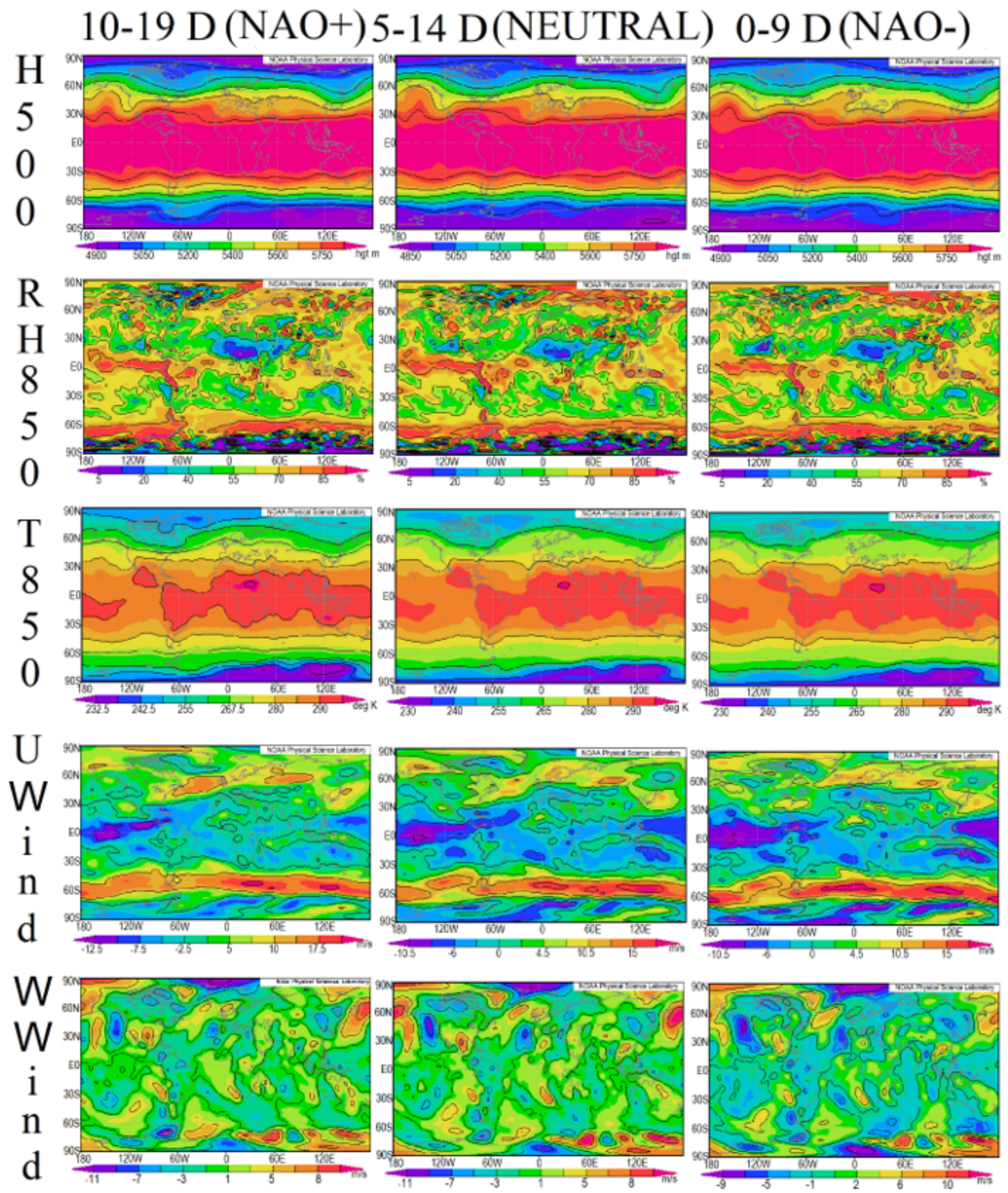

Figure 4

NCEP/NCAR Reanalysis for 3 incubation time periods of SARS-CoV-2 (same as in Fig. 3). H500: geopotential height at level $500 \mathrm{Hpa}$; RH850: relative humidity at level 850 hPa; T850: temperature at level 
$850 \mathrm{hPa}$; U wind/Wwind: zonal/meridional winds at level $850 \mathrm{hPa}$ (plotted from NCEP/NCAR Reanalysis, Physical Sciences Laboratory NOAA)
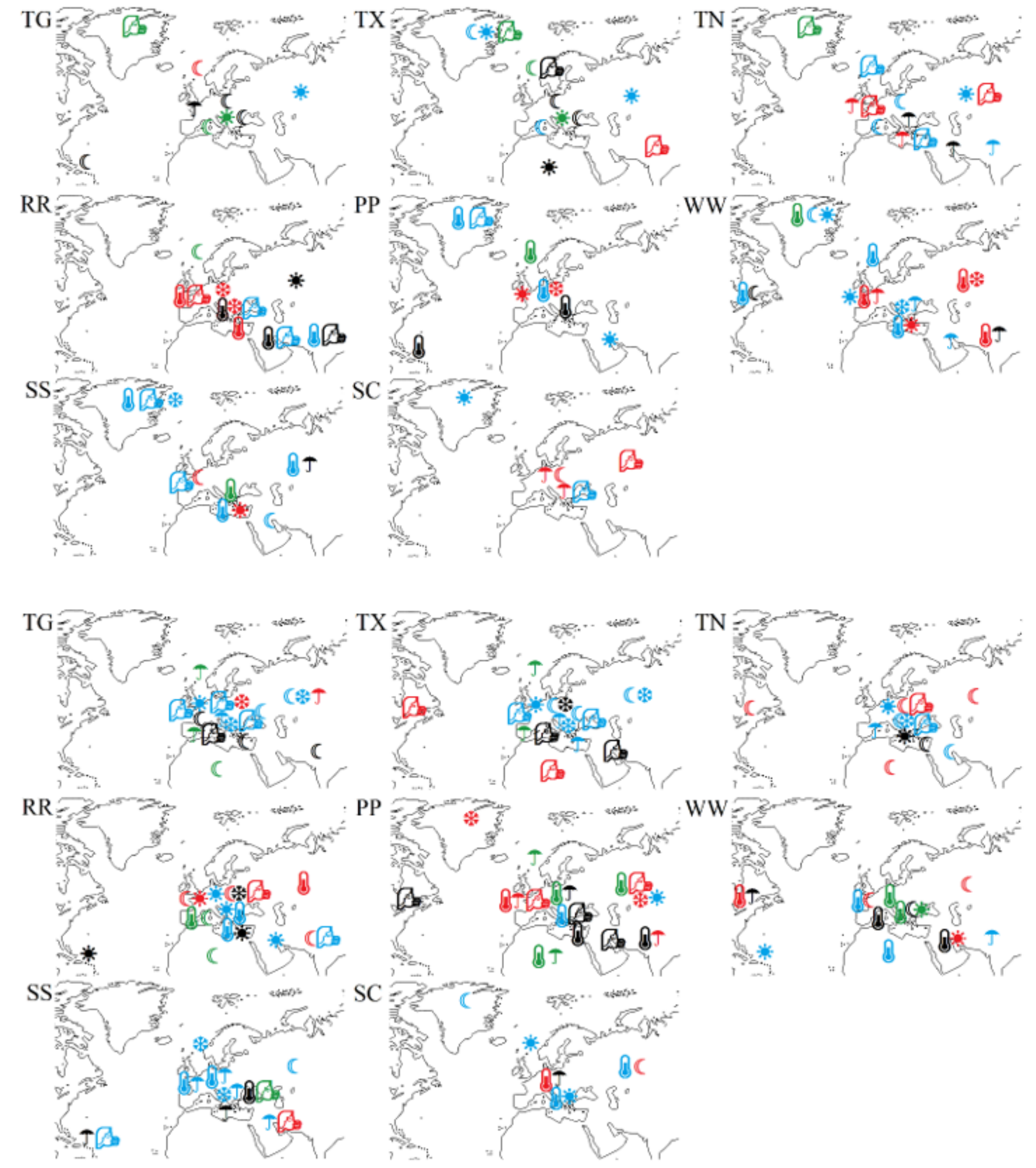

Figure 5

Spearman correlation ranks from Table 3 significant at level 0.10 given to the schematic maps (up -

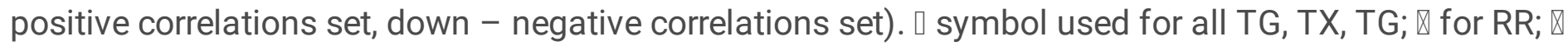
for PP; $\square$ for WW; $₫$ for SS and $₫$ for SC. Blue symbols for NAO- or NAO- and neutral, black symbols for 
neutral, green for $\mathrm{NAO}+$ and NAO- both (or in addition neutral) and red for $\mathrm{NAO}+$ or $\mathrm{NAO}+$ and neutral phase.

\section{Supplementary Files}

This is a list of supplementary files associated with this preprint. Click to download.

- Tables.docx 